\title{
Chronic Discoid Lupus \\ An uncommon cause of nail atrophy
}

"Francisco J. Navarro-Triviño ${ }^{1}$ and Ricardo Ruiz-Villaverde ${ }^{2}$

مرض النئبة المزمنة

فرانسيسكو خوسيه نافارو-تريفينيو و ريكاردو رويز-فيلافيرد

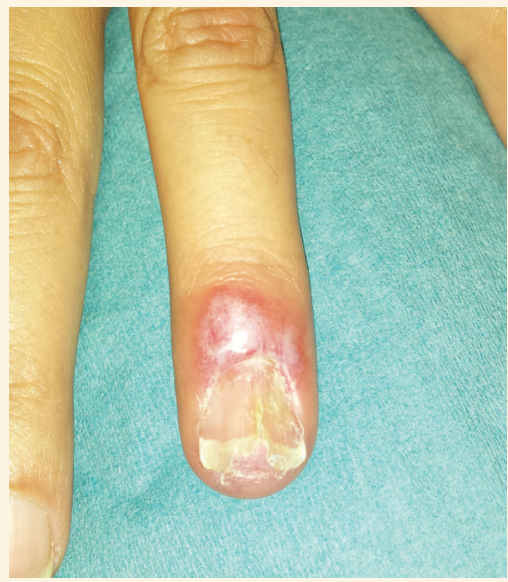

Figure 1: Photograph of the fourth fingernail of the left hand of a 45 year-old woman with discoid lupus erythematosus and Sjögren syndrome showing atrophic changes and whitish cuticles.

A

45 YEAR-OLD WOMAN PRESENTED TO THE outpatient dermatology clinic of the Hospital Alcalá la Real, Jaén, Spain, in 2017 complaining of recent changes to the distal phalanx of the third and fourth finger of her right hand and the fourth finger of her left one. She had a history of discoid lupus erythematosus (DLE) and Sjögren syndrome (SS) without Raynaud's phenomenon. A clinical examination revealed atrophic changes associated with milky-red patches on the affected areas [Figure 1]. The status of the toenails was normal. The patient did not show palmoplantar hyperkeratosis and there was no evidence of mucous membrane ulceration.

The patient underwent a series of laboratory tests which revealed an antinuclear antibody titre of $1 / 640$ with granular and nuclear patterns, anti-SS-related antigen A/ Ro52 level of $>240 \mathrm{U} / \mathrm{mL}$, anti-SS-related antigen B/La level of $>320 \mathrm{U} / \mathrm{mL}$ and rheumatoid factor level of $72.7 \mathrm{UI} / \mathrm{mL}$. However, complement, anti-histidyl transfer ribonucleic acid synthetase, antiscleroderma 70, antiphospholipid and thrombophilia tests were normal.
In addition, thyroid function was normal. Nailfold capillaroscopy was performed using a 3Gen DermLite ${ }^{\circledR}$ dermascope (3Gen Inc., San Juan Capistrano, California, USA) in polarised mode at x10 magnification. This showed dilated and elongated capillaries, increased tortuosities and avascular areas [Figure 2]. These changes were associated with nail pterygium. Onychomycosis was ruled out by a negative dermatophyte culture.

\section{Comment}

First described by Gilliam et al. in 1981, DLE is a chronic scarring skin condition and a common form of cutaneous lupus in European populations. ${ }^{1-3}$ The condition usually has a severe effect on quality of life and emotional wellbeing. ${ }^{2,3}$ Dermoscopy of facial and extra-scalp DLE lesions shows varying features according to disease stage. Erythematous perifollicular whitish 'halos', follicular keratotic plugs, red dots and white scaling are common features in early lesions, while whitish structureless areas and hyperpigmentation (e.g. in a 'honeycomb' or peri- 


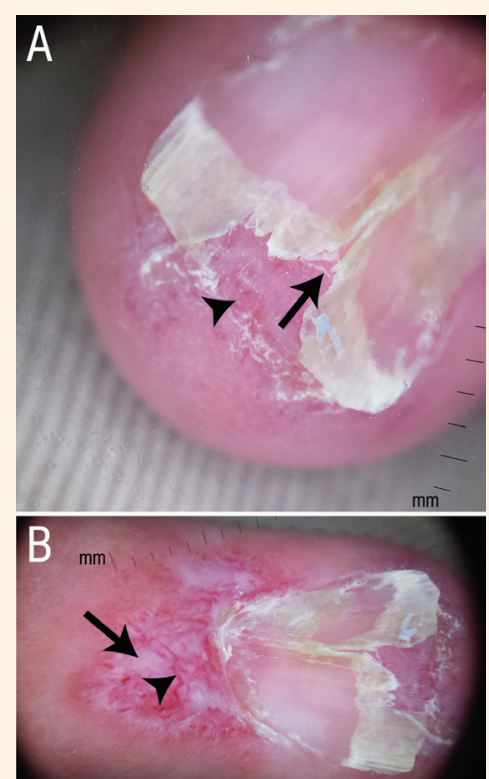

Figure 2: Capillaroscopy images of the fingernail of a 45 year-old woman with discoid lupus erythematosus and Sjögren syndrome showing (A) dystrophic tent-shaped changes in the nail (arrow) as well as distal onycholysis associated with fine superficial desquamation and pointed vessels in the hyponychium (arrowhead) and (B) white avascular areas (arrow) and elongated tortuous capillaries (arrowhead) in the eponychium.

follicular pattern) are frequently observed in late phases. ${ }^{4}$ Ungual involvement is not common. Clinically, DLE usually presents as a well-delimited plaque with red and white areas and is associated with skin and nail trophic changes. ${ }^{5}$

Capillaroscopy is considered the gold-standard technique to evaluate the microvasculature in the proximal area of the nailfold. ${ }^{6}$ Giant capillaries, haemorrhage, avascular areas and neoangiogenesis are considered scleroderma-type features of systemic lupus erythematosus (SLE), rather than DLE. ${ }^{7}$ Correlations have been demonstrated between capillaroscopic abnormalities in SLE and Raynaud's phenomenon, disease activity, endothelial cell activation markers and certain autoantibodies, such as anticardiolipin, anti-U1-ribonucleoprotein, antiSmith and anti-double stranded DNA antibodies. ${ }^{6,8}$

In affected cases where capillaroscopy is not feasible, examination of the nail via routine dermoscopy may provide important information. Although nailfold dermoscopic features may not be specific to DLE, these features have been associated with higher disease activity and internal organ involvement and suggest an important role for chronic microvascular damage in the clinical development of systemic autoimmune disease. ${ }^{7}$ Urowitz et al. described common nail features among patients affected by SLE. ${ }^{9}$ However, little has been published regarding nail trophic changes among patients with DLE.
Nail dystrophy in DLE may allow for the identification of patients with severe conditions and high damage indices. The differential diagnosis of DLE-related nail dystrophy should include onychomycosis-ruled out in the current case-psoriasis, lichen planus, Darier's disease and nail trauma. ${ }^{10}$ A skin biopsy is often not necessary if other discoid skin lesions are present, as in the current case; however, it can be performed in cases wherein the clinical diagnosis is not consistent with DLE.

In terms of topical treatment, clobetasol propionate lacquer, tazarotene cream and tacrolimus ointment may result in a partial therapeutic response. ${ }^{11}$ However, combining this approach with systemic treatment such as methotrexate has produced better response rates. In addition, antiplatelets and/or anticoagulant agents should be considered. ${ }^{5}$

\section{References}

1. Gilliam JN, Sontheimer RD. Distinctive cutaneous subsets in the spectrum of lupus erythematosus. J Am Acad Dermatol 1981; 4:471-5. doi: 10.1016/S0190-9622(81)80261-7.

2. Grönhagen CM, Fored CM, Granath F, Nyberg F. Cutaneous lupus erythematosus and the association with systemic lupus erythematosus: A population-based cohort of 1088 patients in Sweden. Br J Dermatol 2011; 164:1335-41. doi: 10.1111/j.13652133.2011.10272.x

3. Ogunsanya ME, Kalb SJ, Kabaria A, Chen S. A systematic review of patient-reported outcomes in patients with cutaneous lupus erythematosus. Br J Dermatol 2017; 176:52-61. doi: 10.1111/bjd. 14868.

4. Errichetti E, Stinco G. Dermoscopy in general dermatology: A practical overview. Dermatol Ther (Heidelb) 2016; 6:471-507. doi: 10.1007/s13555-016-0141-6.

5. Trüeb RM. Hair and nail involvement in lupus erythematosus. Clin Dermatol 2004; 22:139-47. doi: 10.1016/j.clindermatol.20 03.12.021.

6. Cutolo $\mathrm{M}$, Melsens $\mathrm{K}$, Wijnant $\mathrm{S}$, Ingegnoli $\mathrm{F}$, Thevissen $\mathrm{K}$, De Keyser F, et al. Nailfold capillaroscopy in systemic lupus erythematosus: A systematic review and critical appraisal. Autoimmun Rev 2018; 17:344-52. doi: 10.1016/j.autrev.2017.11.025.

7. Higuera V, Amezcua-Guera LM, Montoya H, Massó F, Patlán M, Paez A, et al. Association of nail dystrophy with accrued damage and capillaroscopic abnormalities in systemic lupus erythematosus. J Clin Rheumatol 2016; 22:13-18. doi: 10.1097/RHU.0000 000000000336

8. Bărbulescu AL, Vreju AF, Bugă AM, Sandu RE, Criveanu C, Tudoraşcu DR, et al. Vascular endothelial growth factor in systemic lupus erythematosus: Correlations with disease activity and nailfold capillaroscopy changes. Rom J Morphol Embryol 2015; 56:1011-16.

9. Urowitz MB, Gladman DD, Chalmers A, Orgyzio MA. Nail lesions in systemic lupus erythematosus. J Rheumatol 1978; 5:441-7.

10. Tully AS, Trayes KP, Studdiford JC. Evaluation of nail abnormalities. Am Fam Physician 2012; 85:779-87.

11. Winkelmann RR, Kim GK, Del Rosso JQ. Treatment of cutaneous lupus erythematosus: Review and assessment of treatment benefits based on Oxford Centre for Evidence-based Medicine criteria. J Clin Aesthet Dermatol 2013; 6:27-38. 